\title{
Microwave-aided transport measurements on high-density two-dimensional electron systems confined at AlGaN/GaN heterointerfaces
}

\author{
D. R. Hang ${ }^{1}$, C. F. Huang ${ }^{2}$, and Y. F. Chen ${ }^{* 1}$ \\ ${ }^{1}$ Department of Physics, National Taiwan University, Taipei, Taiwan 106 \\ 2 National Measurement Laboratory, Centre for Measurement Standards, \\ Industrial Technology Research Institute, Hsinchu, Taiwan 300
}

Received 25 May 2003, accepted 24 June 2003

Published online 20 October 2003

PACS 72.20.My, 73.21.Fg, 73.50.Jt

We present microwave-aided magnetotransport measurements on high-density two-dimensional electron gas (2DEG) systems confined at $\mathrm{AlGaN} / \mathrm{GaN}$ heterointerfaces. We show that the modulated patterns of Shubnikov-de Haas ( $\mathrm{SdH}$ ) oscillations which are due to higher-subband occupation can be drastically enhanced by employing the microwave modulation technique, allowing direct experimental observation and analysis. In addition, the $\mathrm{SdH}$ oscillations can be observed at a much lower magnetic field. Thus we provide a powerful way to study high-density $2 \mathrm{DEG}$ systems which possesses higher resolution and enhanced pattern, does not alter carrier concentrations, and merely requires direct data analysis without numerical artifacts compared with conventional measurements.

(C) 2003 WILEY-VCH Verlag GmbH \& Co. KGaA, Weinheim

In the past several years, fundamental properties of $\mathrm{Al}_{x} \mathrm{Ga}_{1-x} \mathrm{~N} / \mathrm{GaN}$ heterostructures have received a great amount of attention for the application of heterostructure-field-effect transistors (HFETs), which are capable of working at high frequencies in high-power and high-temperature environments [1-3]. They are also very promising for applications in microwave and optoelectronic devices. Compared with other III-V material based HFETs, a larger amount of two-dimensional electron gas (2DEG) can be easily accumulated in GaN-based ones. The lattice mismatch of $2.5 \%$ between AlN and GaN and the lack of inversion symmetry in the wurtzite structure result in large induced and spontaneous polarizations [2]. Therefore the better carrier confinement at $\mathrm{Al}_{x} \mathrm{Ga}_{1-x} \mathrm{~N} / \mathrm{GaN}$ interface than that at $\mathrm{Al}_{x} \mathrm{Ga}_{1-x} \mathrm{As} / \mathrm{GaAs}$ interface arising from the large conduction-band offset [3] and strong piezoelectric polarization of the $\mathrm{Al}_{x} \mathrm{Ga}_{1-x} \mathrm{~N}$ barrier layer naturally leads to a recent research interest in high-carrierdensity $\mathrm{Al}_{x} \mathrm{Ga}_{1-x} \mathrm{~N} / \mathrm{GaN}$ heterostructures in which multiple subbands were occupied $[4,5]$. In order to optimize the performance of HFETs with high electron densities, it is necessary to be able to probe efficiently the subband properties of $2 \mathrm{DEG}$ of high densities at $\mathrm{Al}_{x} \mathrm{Ga}_{1-x} \mathrm{~N} / \mathrm{GaN}$ heterointerfaces.

Shubnikov-de Haas $(\mathrm{SdH})$ measurements are often employed to characterize the properties of 2DEG in different subbands at $\mathrm{Al}_{x} \mathrm{Ga}_{1-x} \mathrm{~N} / \mathrm{GaN}$ heterointerface. When carriers only populate the first subband, the SdH pattern is simple and the analysis is straightforward. As carriers populate the second subband, the SdH pattern has double periodicity and the detailed analysis counts on the effectiveness of numerical methods. In samples with low second-subband carrier population, it is usually difficult to resolve the second periodicity because it is easily obscured by the floating background in the longitudinal resistance $\rho_{x x}(B)$ during fast Fourier transformation. One way to overcome this difficulty is to enhance the sensitiv-

" Corresponding author: e-mail: yfchen@phys.ntu.edu.tw, Phone: +886-2-3366-5125, Fax: +886-2-2363-9984 
ity and effectiveness of conventional magnetotransport measurements. In this paper, we report on microwave-aided transport measurements on high-density 2DEG confined at AlGaN/GaN heterointerfaces. We show that the modulated patterns of SdH oscillations with multi-frequency can be drastically enhanced by employing the microwave modulation technique, making direct experimental observation and analysis feasible.

We performed $\mathrm{SdH}$ measurements on two modulation-doped $\mathrm{Al}_{0.22} \mathrm{Ga}_{0.78} \mathrm{~N} / \mathrm{GaN}$ heterostructures grown by atmospheric pressure metal-organic chemical vapor deposition on the (0001) surface of sapphire substrates. A nucleation $\mathrm{GaN}$ buffer layer was deposited at $488^{\circ} \mathrm{C}$, followed by a unintentionally doped GaN (i-GaN) layer of $2 \mu \mathrm{m}$ thickness grown at $1071^{\circ} \mathrm{C}$. The barrier layer is a Si-doped $\mathrm{Al}_{0.22} \mathrm{Ga}_{0.78} \mathrm{~N}$ (n-AlGaN) layer of thickness $25 \mathrm{~nm}$ for sample A and $30 \mathrm{~nm}$ for sample B. The doping concentration is $1.2 \times 10^{18} \mathrm{~cm}^{-3}$. The one-side doping results in the triangular confinement of carriers in the heterojunctions. Between the $\mathrm{n}-\mathrm{AlGaN}$ and $\mathrm{i}-\mathrm{GaN}$ layer, a 5-nm-thick unintentionally doped $\mathrm{Al}_{0.22} \mathrm{Ga}_{0.78} \mathrm{~N}$ spacer was inserted for sample $\mathrm{A}$ to reduced remote impurity scattering. The samples were placed inside a 6 Tesla Oxford superconducting magnet and immersed in liquid helium. The temperature could be cooled down to as low as $3.6 \mathrm{~K}$. The data were taken by conventional lock-in techniques.

In our studies, we additionally employed a novel technique of $\mathrm{SdH}$ measurement that can drastically enhance the $\mathrm{SdH}$ pattern, which is based on microwave modulation. Here, the measurements were done electrically in phase under microwave modulation. This method has advantages of unchanging carrier concentration and not diminishing modulation pattern compared with other variant techniques like optically modulated $\mathrm{SdH}$ and optically-detected microwave modulated $\mathrm{SdH}$ measurements. In our previous report [6], we have shown that this technique is suitable for studying novel wide band-gap heterostructures where moderate mobilities and heavier effective mass (rapid damping $\mathrm{SdH}$ amplitudes) are frequently encountered. Detailed experimental setup can be found in one of our previous papers [6].

Figures 1 and 2 show $\mathrm{SdH}$ oscillations taken at $3.6 \mathrm{~K}$ for sample $\mathrm{A}$ and sample B, respectively. The two-dimensional characters have been further confirmed by rotating the sample orientation against the magnetic field. The strong double periodicity of the $\mathrm{SdH}$ oscillations is easily recognized for sample $\mathrm{A}$ and also exists in sample B, which will be shown below. The modulation doping and the strong polarization field give rise to a large amount of confined 2DEG in the heterointerface. The electrons start to populate the second subband because of the high 2DEG sheet concentrations at the heterointerfaces, resulting in the double periodicity.

It is straightforward to obtain the carrier concentrations corresponding to the first subband from highfrequency oscillations. The successive oscillation numbers as a function of inverse magnetic fields of the $\mathrm{SdH}$ oscillation minima for both samples are plotted as solid circles in both insets in Fig. 1 and 2. The data can be described by the simple linear equation [2]

$$
\frac{1}{B_{N}}=N \frac{e}{\pi \hbar n}+C
$$

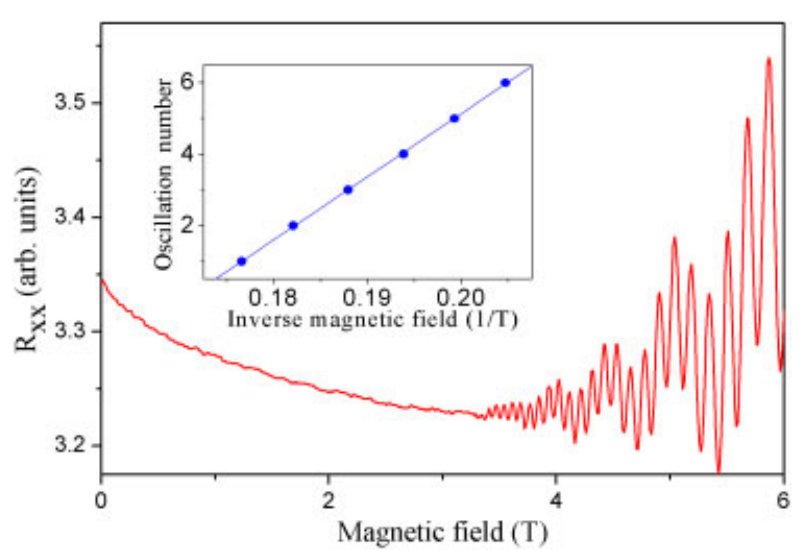

Fig. 1 (online colour at: www.interscience. wiley.com) Magnetoresistance of 2DEG in $\mathrm{Al}_{0.22} \mathrm{Ga}_{0.78} \mathrm{~N} / \mathrm{GaN}$ heterostructures as a function of magnetic field at $3.6 \mathrm{~K}$ in sample A. The inset shows successive oscillation numbers (solid circles) as a function of inverse magnetic fields of the $\mathrm{SdH}$ minimum. 


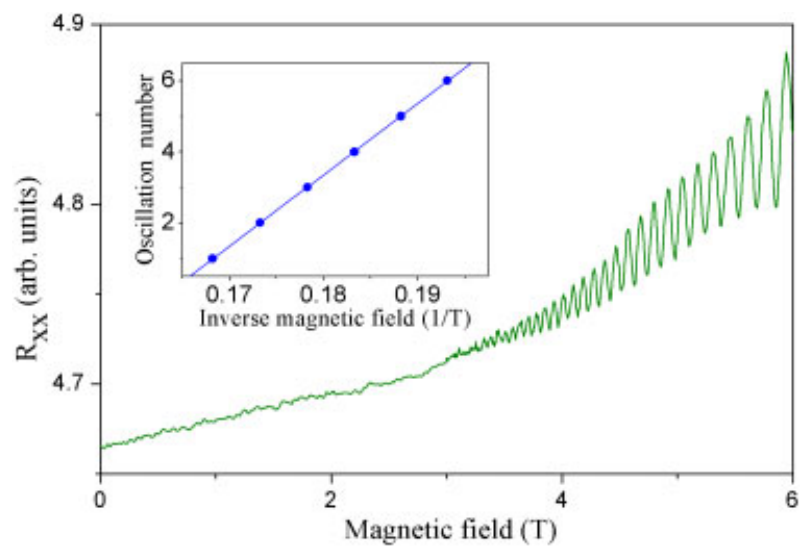

Fig. 2 (online colour at: www.interscience. wiley.com) Magnetoresistance of 2DEG in $\mathrm{Al}_{0.22} \mathrm{Ga}_{0.78} \mathrm{~N} / \mathrm{GaN}$ heterostructures as a function of magnetic field at $3.6 \mathrm{~K}$ in sample $\mathrm{B}$. The carrier concentration can be deduced from the inset.

where $B_{N}$ represents each magnetic field at successive oscillation minimum, $N$ is an integer, $C$ is a constant, and $n$ is the carrier concentration. The choice of the oscillation number is arbitrary. The solid lines in both insets show the fittings to Eq. (1). The slopes of the lines correspond to carrier densities of $8.48 \times 10^{12} \mathrm{~cm}^{-2}$ for sample A and $9.6 \times 10^{12} \mathrm{~cm}^{-2}$ for sample B, respectively.

Figures $3 \mathrm{a}$ and $\mathrm{b}$ display the microwave-modulated $\mathrm{SdH}$ patterns at the same temperature under the modulation of a $3.7 \mathrm{GHz}$ microwave radiation for sample A and sample $\mathrm{B}$, respectively. The SdH patterns are considerably enhanced for both samples. It is quite amazing that the visible signal noise is almost washed out and the onset of $\mathrm{SdH}$ oscillations is noticeably lowered. For the data taken in sample A, it is significant to note that the short-period oscillations die away at magnetic fields lower than 2.5 Tesla, while the long-period oscillations persist. Therefore we provide direct experimental evidence that the 2DEG in the second subband has a higher mobility than that in the first subband in the modulation-doped $\mathrm{Al}_{0.22} \mathrm{Ga}_{0.78} \mathrm{~N} / \mathrm{GaN}$ heterostructures by means of microwave-modulated magnetotransport measurements. On the other hand, the resolution of the conventional $\mathrm{SdH}$ measurements under similar condition is not capable of comparing these oscillations originated from the two-subband occupancy directly. This shows an advantage of the microwave-modulation technique.

From the fit to Eq. (1), the second-subband carrier density in sample A is determined to be $1.78 \times 10^{12} \mathrm{~cm}^{-2}$, and the total 2DEG sheet concentration in the triangular well is $1.02 \times 10^{13} \mathrm{~cm}^{-2}$. Due

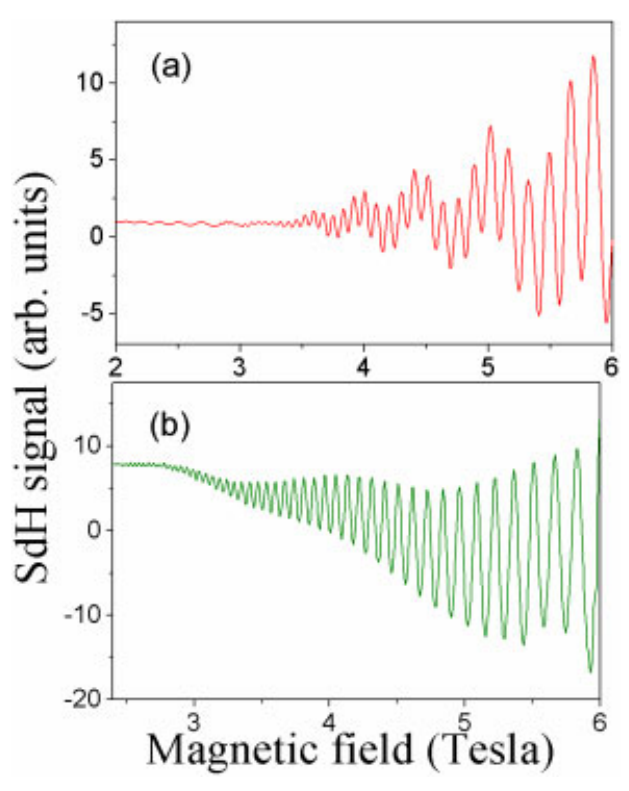
to the long- period modulation, the double periodicity in sample B is not straightforwardly recognizable in conventional $\mathrm{SdH}$ measurement. But the modulated $\mathrm{SdH}$ pattern can be readily identifiable in microwavemodulated $\mathrm{SdH}$ oscillations, as shown in Fig. $3 \mathrm{~b}$. Again, this shows the advantage of the microwavemodulated technique. By the same approach above, the second-subband carrier density is estimated to be

Fig. 3 (online colour at: www.interscience.wiley.com) The electrically detected microwave-modulated $\mathrm{SdH}$ oscillations at temperature $3.6 \mathrm{~K}$ for (a) sample A and (b) sample B. For sample A, long-period oscillations can be clearly observed in the low-field region. 
$4.8 \times 10^{11} \mathrm{~cm}^{-2}$. Thus, the total $2 \mathrm{DEG}$ sheet concentration resides in the heterojunction for sample $\mathrm{B}$ is $1.01 \times 10^{13} \mathrm{~cm}^{-2}$, which is very close to that for sample A.

In conclusion, we present microwave-aided magnetotransport measurements on high-density 2DEG confined at $\mathrm{AlGaN} / \mathrm{GaN}$ heterointerfaces. The second-subband population is manifested by the multifrequency in the SdH oscillations. We demonstrated that the modulated patterns of $\mathrm{SdH}$ oscillations due to second-subband occupancy can be drastically enhanced by employing the microwave modulation technique. In addition, the $\mathrm{SdH}$ oscillations can be observed at a much lower magnetic field. We provide direct experimental evidence that the 2DEG in the second subband has a higher mobility than that in the first subband in the modulation-doped $\mathrm{Al}_{0.22} \mathrm{Ga}_{0.78} \mathrm{~N} / \mathrm{GaN}$ heterostructures by means of microwavemodulated magnetotransport measurements. Therefore we offer a powerful method to study high-density 2DEG systems which possesses higher resolution and merely requires direct data analysis compare with conventional measurements.

Acknowledgements This work was partly supported by the National Science Council and the Ministry of Education of the Republic of China.

\section{References}

[1] D. R. Hang, C.-T. Liang, C. F. Huang, Y. H. Chang, Y. F. Chen, H. X. Jiang, and J. Y. Lin, Appl. Phys. Lett. 79, 66 (2001).

[2] J. A. Garrido, J. L. Sánchez-Rojas, A. Jiménez, E. Muñoz, F. Omnes, and P. Gibart, Appl. Phys. Lett. 75, 2407 (1999).

[3] D. R. Hang, C. H. Chen, Y. F. Chen, H. X. Jiang, and J. Y. Lin , J. Appl. Phys. 90, 1887 (2001).

[4] Z.-F. Li, W. Lu, S. C. Shen, S. Holland, C. M. Hu, D. Heitmann, B. Shen, Y. D. Zheng, T. Someya, and Y. Arakawa, Appl. Phys. Lett. 80, 431 (2002).

[5] Z. W. Zheng, B. Shen, R. Zhang, Y. S. Gui, C. P. Jiang, Z. X. Ma, G. Z. Zheng, S. L. Guo, Y. Shi, P. Han, Y. D. Zheng, T. Someya, and Y. Arakawa, Phys. Rev. B 62, R7739 (2000).

[6] D. R. Hang, C.-T. Liang, J.-R. Juang, T.-Y. Huang, W. K. Hung, Y. F. Chen, G.-H. Kim, Jae-Hoon Lee, and Jung-Hee Lee, J. Appl. Phys. 93, 2055 (2003). 